\title{
Assessment of pain control, quality of life, and predictors of success after gamma knife surgery for the treatment of trigeminal neuralgia
}

\author{
Ajay Jawahar, M.Sc., M.D., Rishi Wadhwa, B.S., Caglar Berk, M.D., \\ Gloria Caldito, Ph.D., Allyson Delaune, R.N., Federico Ampil, M.D., \\ Brian Willis, M.D., Donald SMith, M.D., AND ANIL NANDA, M.D. \\ Departments of Neurosurgery, Biometry, and Radiation Oncology, Louisiana State University Health \\ Sciences Center, Shreveport, Louisiana
}

\begin{abstract}
Object. There are various surgical treatment alternatives for trigeminal neuralgia (TN), but there is no single scale that can be used uniformly to assess and compare one type of intervention with the others. In this study the objectives were to determine factors associated with pain control, pain-free survival, residual pain, and recurrence after gamma knife surgery (GKS) treatment for TN, and to correlate the patients' self-reported quality of life (QOL) and satisfaction with the aforementioned factors.

Methods. Between the years 2000 and 2004, the authors treated 81 patients with medically refractory TN by using GKS. Fifty-two patients responded to a questionnaire regarding pain control, activities of daily living, QOL, and patient satisfaction.

The median follow-up duration was 16.5 months. Twenty-two patients $(42.3 \%)$ had complete pain relief, 14 (26.9\%) had partial but satisfactory pain relief, and in 16 patients $(30.8 \%)$ the treatment failed. Seven patients $(13.5 \%)$ reported a recurrence during the follow-up period, and $25(48.1 \%)$ reported a significant $(>50 \%)$ decrease in their pain within the 1 st month posttreatment. The mean decrease in the total dose of pain medication was $75 \%$. Patients' self-reported QOL scores improved $90 \%$ and the overall patient satisfaction score was $80 \%$.

Conclusions. The authors found that GKS is a minimally invasive and effective procedure that yields a favorable outcome for patients with recurrent or refractory TN. It may also be offered as a first-line surgical modality for any patients with TN who are unsuited or unwilling to undergo microvascular decompression.
\end{abstract}

\section{KEY WoRDS • gamma knife surgery • trigeminal neuralgia • quality of life • neurosurgical outcome}

Trigeminal neuralgia is the most common cranial neuropathy, with a mean incidence of four per 100,000 in the healthy population. ${ }^{12}$ Most patients are treated initially with a variety of medications (for example carbamazepine, phenytoin, gabapentin, and baclofen) up to the maximal dose that they can tolerate. For those who do not receive adequate pain relief from medications or who cannot tolerate the side effects, surgical intervention is a feasible option, although the designation of the best surgical treatment for $\mathrm{TN}$ in the general population remains controversial. ${ }^{7,30,33}$

The description of the first documented case of TN is found in the book Diseases of the Head, written by Dr. Johann Wepfer in 1727; subsequently, Nicholas André de-

Abbreviations used in this paper: $\mathrm{ADL}=$ activities of daily living; EOITP = evaluative overall intensity of total pain experience; GKS = gamma knife surgery; $\mathrm{MR}=$ magnetic resonance; $\mathrm{MVD}=$ microvascular decompression; PPI-VAS = present pain intensity-visual analog scale; $\mathrm{PRI}=$ pain rating index; $\mathrm{QOL}=$ quality of life; $\mathrm{TN}=$ trigeminal neuralgia; $\mathrm{V}_{1}=$ first division of the trigeminal nerve; $\mathrm{V}_{2}=$ second division of the trigeminal nerve; $\mathrm{V}_{3}=$ third division of the trigeminal nerve. scribed two cases of true TN in $1732 .{ }^{4}$ Since then, a variety of treatments have been advocated and practiced by surgeons throughout the world. Various safe and effective surgical treatment alternatives for $\mathrm{TN}$ now exist, such as MVD, ${ }^{46}$ percutaneous radiofrequency rhizotomy, ${ }^{3}$ glycerol rhizolysis ${ }^{14}$ percutaneous balloon microcompression, ${ }^{23}$ and GKS. ${ }^{35}$ All of these surgical procedures have been proposed as treatments of choice for TN in various studies published at different times. ${ }^{24,49}$

None of these studies, however, used uniform outcome measures for success or failure, thus making it difficult for clinicians and patients to determine what treatment may be most efficacious for individual situations. Because pain is both the presenting and the hallmark symptom in TN, the status and severity of the disease at presentation as well as after any procedure largely depend on patients' subjective experience, self-reported QOL, and impairment of ADL due to pain. A variety of factors like age, sex, duration of disease, pain threshold, and, possibly, the emotional makeup of the patient can influence these criteria. Therefore, no single, reliable scale exists in the literature that has gained con- 
A. Jawahar, et al.

sensus as a uniform method that can be used to assess and compare one form of intervention for pain control with another in patients suffering from TN.

It is extremely important that long-term follow-up evaluation should be diligently pursued because $\mathrm{TN}$, a subjective disorder, can often be difficult to assess. The patients may have no pain, slight pain, or moderate pain from one day to the next, and their degree of pain may vary during the course of days, weeks, or years. Most physicians, however, agree that any reliable report should include details about the study design, patient selection criteria, follow-up duration, definitions of the outcome measures, recurrences, successes and failures, details of any complications, and, of course, descriptions of surgical techniques. ${ }^{29}$ We reviewed and stratified our data on the results of GKS treatment for $\mathrm{TN}$ based on these recommended criteria. Our study objectives were to determine factors associated with pain control, pain-free survival, residual pain, and recurrence after GKS treatment for TN and to correlate patients' self-reported QOL and satisfaction with these aforementioned factors.

\section{CLINICAL MATERIAL AND METHODS}

\section{Patient Population}

Between 2000 and 2004, 81 patients with medically refractory TN were treated with GKS by using the Leksell Gamma Knife Model B (Elekta Instruments, Atlanta, GA) at the Gamma Knife Radiosurgery Center of Louisiana State University Health Sciences Center in Shreveport. The follow-up duration was at least 6 months in 68 patients, and these were the ones who were contacted to participate in the study. A questionnaire was mailed to all of them, and subsequent telephone calls were made to solicit their response. Fifty-two patients $(76.5 \%)$ responded to the follow-up questionnaire, two patients had died (one at 6 and one at 7 months after radiosurgery) due to cardiac disease, two did not respond to the questionnaire, and the other 12 could not be contacted.

The study cohort consisted of the 52 patients (18 men [34.6\%] and 34 women [65.4\%]) who responded to a survey regarding the outcome of their GKS treatment. Patients ranged in age from 37 to 88 years, with a median age of 66.5 years. Twenty-seven patients $(51.9 \%)$ had left-sided pain and $25(48.1 \%)$ had pain on the right side. Only one patient (1.9\%) presented with bilateral pain and underwent bilateral GKS. The distribution of pain among the branches of the trigeminal nerve was as follows: four in $\mathrm{V}_{1}, 16$ in $\mathrm{V}_{2}$, 14 in $V_{3}$ (including one patient with bilateral $V_{3}$ pain), six in $V_{1}$ and $V_{2}$, and nine in $V_{2}$ and $V_{3}$. Two patients presented with pain involving all divisions of the trigeminal nerve on the affected side (Table 1).

The pain was clinically characterized as "typical" in 47 patients (90.4\%); atypical features were present in five (9.6\%). The median pain duration was 70 months (range 5-282 months), whereas the median time between establishing the diagnosis of $\mathrm{TN}$ and GKS treatment was 60 months (range 4-240 months).

\section{Patient Selection and Pain Management Criteria}

Patients with electrical shock-like paroxysmal facial pain involving one or more divisions of the trigeminal nerve and
TABLE 1

Side and branches of the trigeminal nerve involved in pain in 52 patients with refractory $T N$ who underwent $G K S$

\begin{tabular}{lrrrccccr}
\hline \hline & \multicolumn{8}{c}{ Branch } \\
\cline { 2 - 7 } Side & $\mathrm{V}_{1}$ & $\mathrm{~V}_{2}$ & $\mathrm{~V}_{3}$ & $\mathrm{~V}_{1}+\mathrm{V}_{2}$ & $\mathrm{~V}_{2}+\mathrm{V}_{3}$ & $\mathrm{~V}_{1}+\mathrm{V}_{3}$ & $\begin{array}{c}\mathrm{V}_{1}+\mathrm{V}_{2} \\
+\mathrm{V}_{3}\end{array}$ & Total \\
\hline $\mathrm{rt}$ & 1 & 9 & 5 & 4 & 3 & 1 & 1 & 24 \\
lt & 3 & 7 & 8 & 2 & 6 & 0 & 1 & 27 \\
bilat & 0 & 0 & 1 & 0 & 0 & 0 & 0 & 1 \\
total & 4 & 16 & 14 & 6 & 9 & 1 & 2 & 52 \\
\hline
\end{tabular}

with no findings of pathological entities on cranial MR imaging or computerized tomography scans were considered to have idiopathic TN. All of our patients had been evaluated using cranial MR imaging prior to referral for GKS to exclude any lesion in the cerebellopontine angle, petrous apex, cavernous sinus, or cranial base, and none had abnormal neurological findings related to the trigeminal nerve at the time of diagnosis.

Because medical treatment is always the first step for the management of TN, all patients in this study had undergone a trial of medications that failed to provide adequate pain control, although they had a good response at earlier stages of their disease. Carbamazepine was the principal medication used before GKS. Twenty-three patients (44.2\%) taking this agent and 12 patients (23.1\%) taking narcotic drugs reported serious side effects like dizziness, difficulty concentrating, drowsiness, nausea, vomiting, and blurred vision, and were unable to increase their medication dose up to a level that might be sufficient for pain relief.

Twenty-six patients $(50 \%)$ in this cohort had previously undergone various surgical interventions and failed to get either adequate or permanent pain relief from those procedures. Our institutional policy is to offer GKS for TN to patients who are older than 65 years (although we do not enforce a strict age limit for MVD), those who do not want MVD, and to those with poor medical status.

\section{Surgical Procedure}

A Leksell model $\mathrm{G}$ stereotactic head frame was affixed to the patient's head after induction of conscious sedation and local anesthesia. One-millimeter-thick, Gd-enhanced axial MR imaging slices of the brain were obtained with the spoiled gradient-recalled acquisition in steady state sequence. It has been reported that in patients who have not undergone previous surgery for TN, blood vessel-fifth cranial nerve contact revealed with high-resolution MR imaging might indicate a particularly favorable response to GKS. ${ }^{18}$ Nevertheless, in a previous study performed at our center ${ }^{17}$ it was concluded that the presence or absence of vascular compression at the trigeminal nerve root entry zone was not a predictor of favorable outcome after GKS, so this factor was not taken into consideration for the current study.

The imaging data were transferred to the Gamma Knife computer workstation via the Ethernet. Leksell Gamma Plan software was used to localize the radiosurgical target. A team composed of a neurosurgeon, a radiation oncologist, and a medical physicist performed dose planning. A 
$50 \%$ isodose line enclosed the target margin receiving the prescribed dose in all patients. A single 4-mm collimator was used to target the trigeminal nerve root entry zone approximately 2 to $4 \mathrm{~mm}$ distal to the nerve's exit from the pons before it enters the Meckel cave. The irradiated tissue volume was $88 \mathrm{~mm}^{3}$ for all patients. The mean maximum prescribed dose was 80 Gy (range 70-90 Gy) and the mean marginal dose was 40 Gy (range 35-45 Gy). During targeting, precautions were taken to prevent the pons and brainstem from receiving more than $15 \mathrm{~Gy}$ at the maximal exposure point. Radiosurgery treatment was administered using the Leksell Gamma Knife model B-2 (Elekta Instruments) before July 2003 and we have used model C since then. At our institution, we follow the policy of administering a single intravenous dose of $40 \mathrm{mg}$ methylprednisolone at the conclusion of the radiosurgery procedure in all the cases to prevent the occurrence of immediate postradiosurgery edema in the normal brain tissue surrounding the target volume, which is unavoidably exposed to fall-off radiation isodoses. Patients were observed overnight at the hospital and discharged home the following day.

\section{Follow-Up Protocol}

Regular follow-up evaluations consisted of clinic visits at 6-month intervals post-GKS. Success of the procedure was assessed subjectively (degree of pain relief) with patient interviews and objectively with a focused neurological examination, looking for any possible complications related to GKS. It has been reported in a previous study that results of enhanced MR imaging at 3 to 6 months after GKS at a maximal dose of 70 to 90 Gy do not correlate with the clinical response, and therefore routine posttreatment MR imaging is not warranted. ${ }^{26}$ We therefore did not include any imaging studies as a part of our routine follow-up protocol.

\section{Data Acquisition and Outcome Parameters}

All data available from previous surgical and clinical follow-up records were reviewed and stored in a computer database. This data set was matched and updated using the information acquired from a detailed questionnaire that included the short-form McGill pain questionnaire, a PPIVAS, select items from the Short Form-36 related to ADL, QOL, impairment due to TN, and a patient satisfaction scale. Together these instruments provided information about the degree and duration of pain relief, the need for further therapy, the presence of any surgical sequelae, and a subjective assessment of the patients' overall degree of improvement regarding ADL and QOL, as well as satisfaction with the outcome. All instruments have already been validated in previous studies. ${ }^{9,28,37,41}$

Parameters to determine pain control were derived from the short-form McGill pain questionnaire. The sensory PRI, affective PRI, total PRI, PPI-VAS, and EOITP were assessed in the patients before and after GKS treatment to determine the amount of pain control. Pain control due to GKS was calculated as either the difference in PPI-VAS or EOITP (sensory PRI + affective PRI), before and after GKS. Data on other patient characteristics were obtained to determine factors associated with pain control and TN recurrence.

\section{Statistical Analysis}

Except for age, the quantitative variables measured in the patients such as pain control measures, QOL and satisfaction scores, pain duration, and radiosurgery prescription dose were distributed nonnormally. Hence, nonparametric methods such as the Spearman rank correlation and Wilcoxon rank-sum tests were used to determine factors significantly correlated with recurrence and pain control. A general linear model was used to determine independent significant factors for pain control. To account for possible correlations among multiple pain-producing branches within patients, the proportion of patients with residual pain in a branch that was a source of the initial pain were compared among the three possible branches for pain by using a general estimating equation method.

The latency period after which the they became free of pain for the first time after GKS was determined for all patients. The Kaplan-Meier method was used to estimate the pain-free survival rate and the latency period to being free of pain. The Cox proportional hazards model was used to determine significant factors for pain-free survival or period to being pain-free. The same survival analyses were performed to determine significant factors for disease-free interval or time to recurrence. A 5\% level of significance was used for all statistical tests, and SAS Version 8.2 software (SAS Institute Inc., Cary, NC) was used for statistical computing.

Because most of the previously published studies regarding outcome of surgical treatment for TN used more global measures to stratify their results, for ease of comparison we also included a similar stratification: complete pain relief (free of pain with no medications); partial, satisfactory pain relief (recurrent or residual pain was less severe than that occurring preoperatively and that pain could be controlled with medication); or treatment failure (had no relief and/or worsening of symptoms or needed a subsequent procedure).

\section{RESULTS}

The patients were followed for a mean period of 16.5 months (range 6-45 months). Thirty-six patients (69.2\%) in the current study experienced complete or satisfactory pain relief at least once after GKS; however, seven of these patients $(19.4 \%)$ had recurrence of pain at various intervals. Overall, 22 patients $(42.3 \%)$ had complete pain relief, 14 $(26.9 \%)$ had partial but satisfactory pain relief, and in 16 $(30.8 \%)$ the treatment failed. Twenty-five patients $(48.1 \%)$ reported a significant $(>50 \%)$ decrease in their pain within the 1st month following GKS.

The mean decrease in the total dose of pain medication was $75 \%$. The mean improvement on PPI-VAS was $49.5 \%$, the patients' self-reported QOL scores improved 90\%, and the overall patient satisfaction was $80 \%$. Table 2 displays in greater detail all the parameters that were used to analyze the outcome for the current study. During the follow-up period, the proportions of residual pain were six $(46.2 \%)$ of $13,15(45.4 \%)$ of 33 , and $15(57.7 \%)$ of 26 for the sixth, seventh, and eighth cranial nerve branches, respectively. Also, a general estimating equation model showed that the chance of having residual pain after GKS did not depend on the branch of the trigeminal nerve that was initially involved with pain $(\mathrm{p}=0.93)$. 
A. Jawahar, et al.

TABLE 2

Quantitative variables in 52 patients with medically refractory $T N$ who underwent $G K S^{*}$

\begin{tabular}{lc}
\hline \multicolumn{1}{c}{ Variable } & Median (range) \\
\hline age (yrs) & $66.5(37-88)$ \\
pain duration (mos) & $70.0(5-282)$ \\
pain duration from diagnosis (mos) & $60.0(4-240)$ \\
FU duration (mos) & $16.5(6-45)$ \\
\% decrease in meds & $75.0(0-100)$ \\
QOL score (scale 1-5) & $4.5(1-5)$ \\
satisfaction score & $4.0(1-5)$ \\
DPPI-VAS (scale 0-100) & $49.5(-6-100)$ \\
$\Delta$ EOITP (scale 0-5) & $3.0(-1-5)$ \\
$\Delta$ S-PRI (scale 0-27) & $9.0(-6-28)$ \\
$\Delta$ A-PRI (scale 0-12) & $2.0(-3-12)$ \\
$\Delta$ T-PRI (scale 0-39) & $10.0(-6-39)$ \\
max radiation dose (Gy) & $80.0(70-90)$ \\
\hline
\end{tabular}

* A-PRI = affective PRI; meds = medications; S-PRI = sensory PRI; TPRI $=$ total PRI.

Correlations among quantitative factors were analyzed using the Spearman rank correlation coefficient. Significant correlations among the different pain control measures proved that the different indices were consistent in measuring pain (Table 3 ). The pain control measures were significantly correlated with QOL and the patients' self-reported satisfaction scores, and these in turn were highly correlated with each other. Age and pain duration had no significant correlations with pain control, QOL, or patients' self-reported satisfaction scores.

Table 4 shows significant group comparisons for pain control between those with and without residual pain as well as between those with and without a decrease in pain within 1 month after GKS. It also shows significant comparisons in pain duration between those with and without previous procedures. Those without residual pain and with a significant decrease in pain after less than 1 month had significantly better pain control, and those without a previous procedure had a significantly shorter duration of pain. There were no observed significant differences in pain control or duration for the categories of sex, side of pain, number of branches involved, recurrence, and branch. Hence, these were not significantly associated with pain control or duration.
TABLE 3

Significant correlations among quantitative variables

\begin{tabular}{llcr}
\hline \hline $\begin{array}{c}\text { Variable } \\
\text { Correlated }\end{array}$ & \multicolumn{1}{c}{ w/ Variable } & $\begin{array}{c}\text { Correlation } \\
\text { Coefficient }\end{array}$ & p Value \\
\hline$\Delta$ VAS & $\Delta$ EOITP & 0.891 & $<0.01$ \\
& $\Delta$ T-PRI & 0.766 & $<0.01$ \\
& $\Delta$ S-PRI & 0.747 & $<0.01$ \\
& $\Delta$ A-PRI & 0.665 & $<0.01$ \\
& QOL & 0.649 & $<0.01$ \\
$\Delta$ EOITP & satisfaction score & 0.628 & $<0.01$ \\
& $\Delta$ S-PRI & 0.757 & $<0.01$ \\
& $\Delta$ A-PRI & 0.636 & $<0.01$ \\
$\Delta$ QO-PRI & satisfaction score & 0.668 & $<0.01$ \\
& $\Delta$ A-PRI & 0.636 & $<0.01$ \\
& QOL & 0.743 & $<0.01$ \\
QOL & satisfaction score & 0.613 & $<0.01$ \\
& satisfaction score & 0.586 & $<0.01$ \\
& & 0.781 & $<0.01$ \\
\hline
\end{tabular}

To determine independent, significant factors for pain control, defined as either the change in PPI-VAS or EOITP, a multivariate model using the change in these parameters as the dependent variable and the factors observed to be significantly associated with pain control from individual univariate analyses (QOL, overall patient satisfaction score, presence of residual pain, and pain decrease within 1 month after GKS) was constructed from the data. Given the high correlation between QOL and overall patient satisfaction scores and between the presence of residual pain and a decrease in pain within 1 month after GKS, the only independent, significant predictors for pain control were the QOL score and the presence of residual pain. The results of the multivariate analysis are shown in Table 5.

Figure 1A shows the proportion of patients who were not yet free of pain at different times after GKS treatment, and Fig. 1B shows the proportion of patients without recurrence at different follow-up times after GKS. The median time to pain control after GKS was 9 months and the median interval at which patients remained pain-free was 17 months (95\% confidence interval 10-23 months) after GKS. The recurrence rate was estimated to be $23.1 \%$ and the median time to recurrence was 17.5 months after GKS treatment.

Using separate univariate analyses, the factors significantly associated with first time to reaching pain-free status

TABLE 4

Significant group comparisons on pain control measures

\begin{tabular}{|c|c|c|c|c|}
\hline Pain Control/Duration & Group & No. of Patients & Median (range) & $\mathrm{p}$ Value \\
\hline \multirow[t]{2}{*}{$\triangle \mathrm{PPI}-\mathrm{VAS}$} & w/o residual pain & 22 & $92.5(-3-100)$ & \multirow[t]{2}{*}{$<0.01$} \\
\hline & w/ residual pain & 30 & $24(-6-82)$ & \\
\hline \multirow[t]{2}{*}{$\triangle \mathrm{EOITP}$} & w/o residual pain & 22 & $5(1-5)$ & \multirow[t]{2}{*}{$<0.01$} \\
\hline & w/ residual pain & 30 & $1.5(-1-5)$ & \\
\hline \multirow[t]{2}{*}{$\triangle \mathrm{PPI}-\mathrm{VAS}$} & w/o pain decrease in $<1 \mathrm{mo}$ & 27 & $31(-6-96)$ & 0.016 \\
\hline & w/ pain decrease in $<1 \mathrm{mo}$ & 25 & $59(-3-100)$ & 0.034 \\
\hline \multirow[t]{2}{*}{$\triangle \mathrm{EOITP}$} & w/o pain decrease in $<1 \mathrm{mo}$ & 27 & $2(-1-5)$ & \multirow[t]{4}{*}{0.024} \\
\hline & $\mathrm{w} /$ pain decrease in $<1 \mathrm{mo}$ & 25 & $3(0-5)$ & \\
\hline \multirow[t]{2}{*}{ duration of pain from diagnosis } & $\mathrm{w} /$ previous procedure & 26 & $62(4-240)$ & \\
\hline & w/ no previous procedure & 26 & $39.5(4-160)$ & \\
\hline \multirow[t]{2}{*}{ duration of pain } & $\mathrm{w} /$ previous procedure & 26 & $96(9-282)$ & \multirow[t]{2}{*}{$<0.01$} \\
\hline & $\mathrm{w} /$ no previous procedure & 26 & $51(5-167)$ & \\
\hline
\end{tabular}



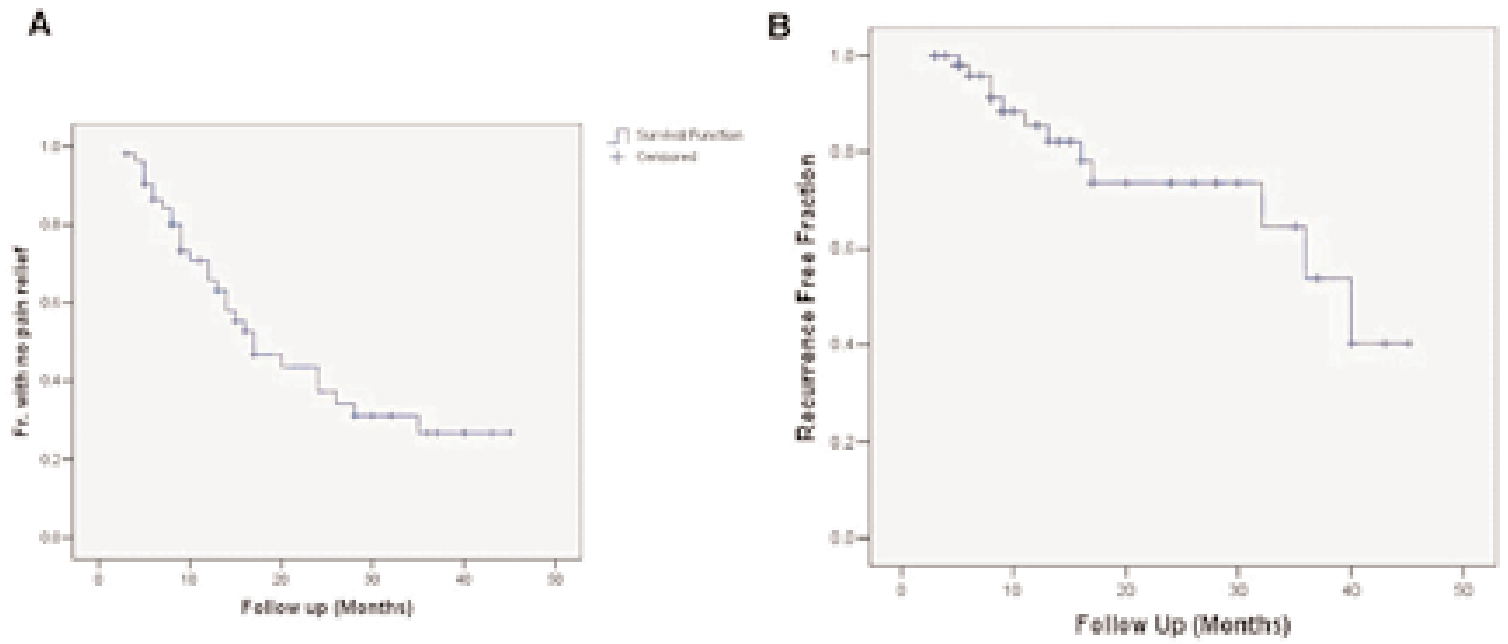

Fig. 1. A: Graph showing pain-free survival curve. Abbreviation: $\mathrm{Fr}=$ fraction.

B: Graph showing recurrence-free survival curve.

are listed in Table 6. Using a Cox proportional hazards model for first time to pain-free status and a stepwise selection of variables among those listed in Table 6 , the only independent, significant predictors of the first time pain-free status would be reached were the QOL score of a patient ( $\mathrm{p}$ $=0.025)$ and change in PPI-VAS $(p<0.01)$, which are shown in Table 7 . There were no significant predictors for recurrence.

\section{DISCUSSION}

Trigeminal neuralgia is the most frequently encountered type of craniofacial neuralgia. It is usually the foremost consideration in the differential diagnosis when a patient reports intermittent, paroxysmal pain confined to the distribution of the trigeminal nerve. Pain may be confined to a small area or spread throughout the distribution of one or more divisions of the trigeminal nerve. ${ }^{2,12}$ The features of the pain that characterize $\mathrm{TN}$, other than its location, are its severity and its lancinating or electrical shock-like quality. The pain can be spontaneous or triggered by almost any stimulus. ${ }^{6,47}$ Many procedures have been devised in an attempt to alleviate $\mathrm{TN}$ in patients in whom medical treatment failed to control pain.

As can be expected, most authors have advocated the superiority of the procedure that they use; hence, large series documenting a considerable number of patients have been published during the last 30 years. ${ }^{26}$ The cause of idiopathic TN has not been fully explained, although compression

TABLE 5

Independent, significant predictors of pain control measures in patients with refractory $T N$

\begin{tabular}{clc}
\hline \hline Pain Control Measure & Predictor & $\mathrm{p}$ Value \\
\hline \multirow{2}{*}{ PPI-VAS } & residual pain & $<0.01$ \\
& QOL score & $<0.01$ \\
\multirow{2}{*}{ EOITP } & residual pain & $<0.01$ \\
& QOL score & $<0.01$ \\
\hline
\end{tabular}

of the trigeminal nerve by a vascular structure (as first observed by Dandy) is the most common finding during surgical exploration of the cerebellopontine angle, and pain is usually relieved after the patient undergoes MVD. ${ }^{36,38,44,50}$ Therefore, MVD has been considered the most appropriate surgical intervention for pain control in TN. ${ }^{10,11}$ The initial success rate has been reported as $92.7 \%$, with mortality rates as low as $0.7 \%$ in larger published series. ${ }^{1}$ In some series, the mean time to recurrence was reported as 1.9 years, with 47 to $75 \%$ of recurrences occurring in the 1st year, and the likelihood of recurrence thereafter was $2 \%$ per year., ${ }^{1,21}$ $, 31,49$ In another series, major recurrences averaged $3.5 \%$ and minor recurrences averaged $1.5 \%$ annually. ${ }^{19}$

Even with high success rates, surgical complications such as cerebellar infarction, cerebrospinal fluid leakage, and pulmonary complications have been observed at a rate of less than $1 \%$, whereas palsies of the seventh and eighth cranial nerves were also noted as unique complications after MVD, occurring in approximately $4 \%$ of patients. ${ }^{52}$ Prevention of complications is extremely important because although TN itself is eminently treatable, deafferentation pain (as a complication of destruction of the trigeminal system) is virtually untreatable. The absence of vascular compression during exploration of the cerebellopontine angle is another critical issue because approxi-

TABLE 6

Significant predictors of first time reaching pain-free status (univariate analyses)

\begin{tabular}{lc}
\hline \multicolumn{1}{c}{ Factor } & $\mathrm{p}$ Value \\
\hline$\Delta$ S-PRI & 0.011 \\
$\Delta$ A-PRI & $<0.01$ \\
$\Delta$ T-PRI & $<0.01$ \\
$\Delta$ PPI-VAS & $<0.01$ \\
DEOITP & $<0.01$ \\
QOL score & $<0.01$ \\
satisfaction score & 0.013 \\
$\%$ decrease in meds & $<0.01$ \\
\hline
\end{tabular}


TABLE 7

Independent, significant predictors of first time reaching pain-free status (multivariate analysis)

\begin{tabular}{cc}
\hline \hline Factor & $\mathrm{p}$ Value \\
\hline$\Delta$ PPI-VAS & $<0.01$ \\
QOL & 0.025 \\
\hline
\end{tabular}

mately $15 \%$ of patients may not have a detectable vascular compression. ${ }^{49}$ Patients with anomalies of the posterior fossa and bone structure variations present another serious challenge during exploration. ${ }^{37}$ Therefore MVD, although it continues to be accepted as the intervention of choice for medically refractory TN by a vast majority of neurosurgeons, has also been subjected to a lot of skepticism, thus mandating the search for other less invasive procedures.

Within the past decade, stereotactic radiosurgery has been proposed as a therapeutic modality for patients with $\mathrm{TN}, 8,43,48,52$ although its exact mechanism of action is still unclear. ${ }^{22,45}$ Most reports in which the outcomes of radiosurgery for $\mathrm{TN}$ are assessed have inadequately addressed the issue of patients' QOL after treatment. ${ }^{42}$ Some authors have reported an overall response with GKS that is similar to that obtained with other surgical therapies performed as the first procedure for the treatment of TN. ${ }^{16}$ Nevertheless, there is no consensus regarding the factors that may influence the favorable outcome. Some say that GKS is among appropriate options for TN for patients in whom optimal medical management, with or without prior invasive treatment, has failed. ${ }^{20}$ Others have proposed that patients with no previous surgical interventions have a better chance of complete pain relief after GKS. ${ }^{13,25,34,42,48}$ Some authors have also reported that patients with an atypical pain component had a lower rate of pain control after GKS..$^{20,35}$ In our study we did not identify a significant difference in pain relief for patients who had undergone previous interventions compared with those receiving radiosurgery as the primary intervention.

It has been asserted that the long-term outcome for secondary TN after GKS is very similar to the outcome for cases of idiopathic TN treated with the same modality and that GKS is safe and effective in both idiopathic and secondary $\mathrm{TN}$, particularly in patients with inoperable lesions. ${ }^{32,51}$ The results of GKS treatment for patients with postherpetic neuralgia are also reported to be satisfactory, and this was corroborated in our study. ${ }^{39}$

A strong correlation between the development of new facial sensory loss and achievement and maintenance of pain relief after GKS has been reported,$^{34}$ although contradicting reports also exist. ${ }^{52}$ We did not find a significant association between pain relief and the development of a new sensory deficit after GKS.

The duration of pain preceding the treatment $(<50$ months) has also been proposed to have an influence on favorable outcome after GKS; ${ }^{43}$ thus, early GKS (once the diagnosis is established) is recommended for patients with TN ${ }^{48}$ In our cohort, we found no significant association between pain duration and favorable outcome.

We used a mean maximal radiosurgery prescription dose of $80 \mathrm{~Gy}(70-90 \mathrm{~Gy})$. It has been reported that a maximal dose of more than $70 \mathrm{~Gy}$ is associated with a significantly greater chance of pain relief, ${ }^{40}$ and radiosurgery prescription doses up to 90 Gy have been shown to be safe. ${ }^{14}$ Within the given dose limits, our statistical analysis did not show a correlation between the maximal radiosurgery prescription dose and good pain control.

In a previous study, patients with sustained pain relief reported a mean $100 \%$ improvement in their QOL as a direct result of pain relief after GKS, and $100 \%$ believed that the procedure was successful, whereas $65 \%$ thought their treatment was a success after a mean $80 \%$ improvement in QOL. ${ }^{42}$ We found similar results; the improvement in QOL and patient satisfaction correlated well with pain control measures. Several measures used to quantify the pain also correlated well with each other, which further supports the reliability of our findings. We were not able to demonstrate any significant association between pain control or recurrence and patients' age, sex, the side and branch of the trigeminal nerve involved in pain, and the radiosurgery prescription dose.

To summarize, there is no single, standardized protocol for treating medically refractory TN. The most important aspects in selecting the procedure are the suitability to the patients' individual condition, their expectations and willingness to take any risks associated with each particular treatment, and the surgeon's comfort level and experience with a particular procedure.

\section{CONCLUSIONS}

Gamma knife surgery is a minimally invasive and effective procedure that may be indicated for TN of both primary and secondary origin, with a favorable outcome that is comparable to the results obtained with MVD. There are still not enough long-term follow-up data available from large series. Considering the satisfactory results in our own experience, GKS may be offered as a feasible modality for patients with refractory TN, provided that the patient clearly understands the advantages and disadvantages of each and every treatment option available.

\section{References}

1. Apfelbaum RI: Advantages and disadvantages of various techniques to treat trigeminal neuralgia, in Rovit RL, Murali R, Jannetta PJ (eds): Trigeminal Neuralgia. Baltimore: Williams \& Wilkins, 1990, pp 239-250

2. Apfelbaum RI: Neurovascular decompression: the procedure of choice? Clin Neurosurg 46:473-498, 2000

3. Barker FG, Jannetta PJ, Bissonette DJ, et al: The long-term outcome of microvascular decompression for trigeminal neuralgia. N Engl J Med 334:1077-1083, 1996

4. Bederson JB, Wilson CB: Evaluation of microvascular decompression and partial sensory rhizotomy in 252 cases of trigeminal neuralgia. J Neurosurg 71:359-367, 1989

5. Berk C, Honey CR: Microvascular decompression for trigeminal neuralgia in patients over 65 years of age. Br J Neurosurg 15:76, 2001 (Letter)

6. Brisman R: Gamma knife radiosurgery for primary management for trigeminal neuralgia. J Neurosurg (Suppl 3) 93: 159-161, 2000

7. Brisman R, Khandji AG, Mooij RBM: Trigeminal nerve-blood vessel relationship as revealed by high-resolution magnetic resonance imaging and its effect on pain after gamma knife radiosurgery for trigeminal neuralgia. Neurosurgery 50: $1261-1267,2002$ 
8. Brown JA, McDaniel MD, Weaver MT: Percutaneous trigeminal nerve compression for treatment of neuralgia: results in 50 patients. Neurosurgery 32:570-573, 1993

9. Burchiel KJ: Facial pain syndromes. Clin Neurosurg 46: 435-445, 2000

10. Burchiel KJ, Clarke H, Haglund M, et al: Long-term efficacy of microvascular decompression in trigeminal neuralgia. J Neurosurg 69:35-38, 1988

11. Burchiel KJ, Slavin KV: On the natural history of trigeminal neuralgia. Neurosurgery 46:152-155, 2000

12. Chang JW, Chang JH, Park YG, et al: Gamma knife radiosurgery for idiopathic and secondary trigeminal neuralgia. J Neurosurg 93 (Suppl 3): 147-151, 2000

13. Dudgeon D, Raubertas RF, Rosenthal SN: The short-form McGill pain questionnaire in chronic cancer pain. J Pain Symptom Manage 8:191-195, 1993

14. Friedman DP, Morales RE, Goldman HW: Role of enhanced MRI in the follow-up of patients with medically refractory trigeminal neuralgia undergoing stereotactic radiosurgery using the gamma knife: initial experience. J Comput Assist Tomogr 25:727-732, 2001

15. Gardner WJ: Trigeminal neuralgia. Clin Neurosurg 15:1-56, 1968

16. Häkanson S: Trigeminal neuralgia treated by the injection of glycerol into the trigeminal cistern. Neurosurgery 9:638-646, 1981

17. Jannetta PJ: Arterial compression of the trigeminal nerve at the pons in patients with trigeminal neuralgia. J Neurosurg 26: 159-162, 1967

18. Jannetta PJ: Microvascular decompression of the trigeminal nerve root entry zone, in Rovit RL, Murali R, Jannetta PJ (eds): Trigeminal Neuralgia. Baltimore: Williams \& Wilkins, 1990, pp 201-222

19. Kamiryo T, Kassell NF, Thai QA, et al: Histological changes in the normal rat brain after gamma irradiation. Acta Neurochir 138:451-459, 1996

20. Kanpolat Y, Savas A, Bekar A, et al: Percutaneous controlled radiofrequency trigeminal rhizotomy for the treatment of idiopathic trigeminal neuralgia: 25 -year experience with 1,600 patients. Neurosurgery 48:524-534, 2001

21. Kao MC: Gamma knife surgery for trigeminal neuralgia. J Neurosurg 96:160-161, 2002

22. Kaye AH: Trigeminal neuralgia: vascular compression theory. Clin Neurosurg 46:499-506, 2000

23. Kondziolka D, Flickinger JC, Lundsford LD, et al: Trigeminal neuralgia radiosurgery: the University of Pittsburgh experience. Stereotact Funct Neurosurg 66 (Suppl 1):343-348, 1996

24. Kondziolka D, Lundsford LD, Flickinger JC: Gamma knife radiosurgery as the first surgery for trigeminal neuralgia. Stereotact Funct Neurosurg 70 (Suppl 1):187-191, 1998

25. Kondziolka D, Lunsford LD, Habeck M, et al: Gamma knife radiosurgery for trigeminal neuralgia. Neurosurg Clin N Am 8: 79-85, 1997

26. Kondziolka D, Perez B, Flickinger JC, et al: Gamma knife radiosurgery for trigeminal neuralgia: results and expectations. Arch Neurol 55:1524-1529, 1998

27. Lichtor T, Mullan JF: A 10-year follow-up review of percutaneous microcompression of the trigeminal ganglion. J Neurosurg 72:49-54, 1990

28. Maesewa S, Salame C, Flickinger JC, et al: Clinical outcomes after stereotactic radiosurgery for idiopathic trigeminal neuralgia. J Neurosurg 94:14-20, 2001

29. Maxwell RE: Clinical diagnosis of trigeminal neuralgia and differential diagnosis of facial pain, in Rovit RL, Murali R, Jannetta PJ (eds): Trigeminal Neuralgia. Baltimore: Williams \& Wilkins, 1990, pp 53-77

30. McHorney CA, Ware JE Jr, Lu JF, et al: The MOS 36-item ShortForm Health Survey (SF-36): III. Tests of data quality, scaling assumptions, and reliability across diverse patient groups. Med Care 32:40-66, 1994
31. McLaughlin MR, Jannetta PJ, Clyde BL, et al: Microvascular decompression of cranial nerves: lessons learned after 4400 operations. J Neurosurg 90:1-8, 1999

32. Melzack R: The McGill Pain Questionnaire: major properties and scoring methods. Pain 1:277-299, 1975

33. Nicol B, Regine WF, Courtney C, et al: Gamma knife radiosurgery using 90 Gy for trigeminal neuralgia. J Neurosurg 93 (Suppl 3):152-154, 2000

34 Nugent GR: Surgical treatment: radiofrequency gangliolysis and rhizotomy, in Fromm GH, Sessle BJ (eds): Trigeminal Neuralgia: Current Concepts Regarding Pathogenesis and Treatment. Boston: Butterworth-Heinemann, 1991, pp 159-184

35. Petit JH, Herman JM, Nagda S, et al: Radiosurgical treatment of trigeminal neuralgia: evaluating quality of life and treatment outcomes. Int J Radiat Oncol Biol Phys 56:1147-1153, 2003

36. Pollock Be, Phuong LK, Gorman DA, et al: Stereotactic radiosurgery for idiopathic trigeminal neuralgia. J Neurosurg 97: 347-353, 2002

37. Regis J, Metellus P, Durfour H, et al: Long-term outcome after gamma knife surgery for secondary trigeminal neuralgia. J Neurosurg 95:199-205, 2001

38. Rogers CL, Shetter AG, Fiedler JA, et al: Gamma knife radiosurgery for trigeminal neuralgia: the initial experience of the Barrow Neurological Institute. Int J Radiat Oncol Biol Phys 47:1013-1019, 2000

39. Rothman KJ, Wepsic JG: Side of facial pain in trigeminal neuralgia. J Neurosurg 40:514-516, 1974

40. Sharp CD, Jawahar A, Warren AC, et al: Gamma knife irradiation increases cerebral endothelial expression of intracellular adhesion molecule 1 and E-selectin. Neurosurgery 53:154-161, 2003

41. Shaya M, Jawahar A, Caldito G, et al: Gamma knife radiosurgery for trigeminal neuralgia: a study of predictors of success, efficacy, safety, and outcome at LSUHSC. Surg Neurol 61:529-535, 2004

42. Shmueli A: The relationship between the visual analog scale and the SF-36 scales in the general population: an update. Med Decis Making 24:61-63, 2004

43. Sindou M, Chiha M, Mertens P: Anatomical finding in microsurgical vascular decompression for trigeminal neuralgia: correlations between topography of pain and site of the neuro-vascular conflict. Acta Neurochir Suppl 64:125-127, 1995

44. Sweet WH: The history of the development of treatment for trigeminal neuralgia. Clin Neurosurg 32:294-318, 1985

45. Taha JM, Tew JM Jr: Comparison of surgical treatments for trigeminal neuralgia: reevaluation of radiofrequency rhizotomy. Neurosurgery 38:865-871, 1996

46. Urgosik D, Vymazal J, Vladyka V, et al: Treatment of postherpetic trigeminal neuralgia with the gamma knife. J Neurosurg 93 (Suppl 3): 165-168, 2000

47. Young RF, Vermeulen SS, Grimm P, et al: Gamma knife radiosurgery for treatment of trigeminal neuralgia: idiopathic and tumor related. Neurology 48:608-614, 1997

48. Young RF, Vermulen S, Posewitz A: Gamma knife radiosurgery for treatment of trigeminal neuralgia. Stereotact Funct Neurosurg 70 (Suppl 1):192-199, 1998

49. Zakrzewska JM, Lopez BC: Quality of reporting in evaluations of surgical treatment of trigeminal neuralgia: recommendations for future reports. Neurosurgery 53:110-122, 2003

Manuscript received March 15, 2005.

Accepted in final form April 8, 2005.

Address reprint requests to: Ajay Jawahar, M.Sc., M.D., Department of Neurosurgery, Louisiana State Health Sciences Center in Shreveport, 1501 Kings Highway, PO Box 33932, Shreveport, Louisiana 71130-3932. email: ananda@1suhsc.edusswamy@1su hsc.edu. 